\title{
Comparative Studies of Covering Rough Set Models
}

\author{
Zhaohao Wang \\ College of Mathematics and Computer Science, Shanxi Normal University, Linfen 041004, China \\ Correspondence should be addressed to Zhaohao Wang; wzhao2019@126.com \\ Received 28 April 2014; Accepted 5 August 2014; Published 28 August 2014 \\ Academic Editor: Roque J. Saltarén
}

Copyright (C) 2014 Zhaohao Wang. This is an open access article distributed under the Creative Commons Attribution License, which permits unrestricted use, distribution, and reproduction in any medium, provided the original work is properly cited.

\begin{abstract}
Many different proposals exist for the definition of lower and upper approximation operators in covering-based rough sets and so many different covering rough set models are built correspondingly. It is meaningful to explore the connection of these covering rough set models for their applications in practice. In this paper, we establish relationships between the most commonly used operators in covering rough set models. We investigate the conditions under which two types of upper approximation operations are identical.
\end{abstract}

\section{Introduction}

Rough set theory is a useful tool for dealing with the vagueness, granularity, and uncertainty in information systems and is also a data mining tool. It was proposed by Pawlak in the early 1980 s $[1,2]$. Since then we have witnessed a systematic, world-wide growth of interest in rough set theory [3-11] and its applications [12-17]. Nowadays, it turns out that this approach is of fundamental importance to artificial intelligence and cognitive sciences, especially in the areas of data mining, machine learning, decision analysis, knowledge management, expert systems, and pattern recognition.

The classical rough set theory is based on equivalence relations, but in some situations, equivalence relations are not suitable for coping with granularity, and thus many practical data sets cannot be handled well. In light of this, equivalence relation has been generalized to similarity relation [18], tolerance relation [19-21], and even arbitrary binary relation [2225 ] in some extensions of the classical rough sets. Another approach is the relaxation of the partition arising from equivalence relation to a covering. The covering of a universe is used to construct the lower and upper approximations of any subset of the universe $[3,6,8,11,26]$.

In [27], Yang and Li presented a summary of seven pairs of approximation operators used by Bonikowski et al. [3], Pomykała [6], Tsang et al. [28], Zhu [29], Zhu and Wang [30], and Xu and Zhang [31]. In particular, Bonikowski et al. first extended Pawlak rough set theory from a partition to a covering in [3]; The second type of covering rough set model was presented by Pomykała in [6], while Tsang et al. [28] studied the third type; $\mathrm{Xu}$ and Wang gave the definition of the sixth type in [32]; Zhu defined the fourth and the fifth types of covering-based approximation in $[29,30]$; The seventh type of approximation operations can be found in [31]. For the seven types of covering rough sets, their basic properties and axiomization problem were studied in $[6,28-32]$, their redundancy problem were considered in $[11,27]$, and their topological properties were explored in [33]. Therefore, these seven pairs of approximation operators play an important role in the study of covering rough set models [34]. It is necessary to explore the relationships among the seven types of covering rough sets. Through the comparison of approximation operations, we can find the difference and relationship between them and give the classification of covering rough sets, which can help a decision maker to choose a suitable rough set model for data analysis. Zhu did some research on the relationships among four types of covering rough sets $[30,35]$. However, Zhu considered only the case that the covering is finite. When we do not restrict the covering to the finite, that is, the covering can be infinite, the corresponding problems become complex. In this paper, we do not limit the covering to the finite and investigate the relationships among seven types of covering rough sets.

The remainder of the paper is organized as follows: Section 2 reviews some basic concepts about covering rough set model. In Section 3, we present some results about 
relationships between the first type of covering rough sets and the second, the third, the fourth, the fifth, the sixth, and the seventh types, and give the conditions under which two types of upper approximation operations are identical. Similarly, in Section 4, we investigate the relationships between the second type of covering rough sets and the third, the fourth, the fifth, the sixth, and the seventh types. In Section 5, we do some research on the relationships between the third type of covering rough sets and the fourth, the fifth, the sixth, and the seventh types. In Section 6, we explore the relationships between the fourth type of covering rough sets and the fifth, the sixth, and the seventh types. In Section 7, we present some results about relationships among the fifth, the sixth, and the seventh types of covering rough sets.

\section{Preliminaries}

In this section, we briefly introduce the basic ideas of rough sets and coverings.

Definition 1 (see [3], covering). Let $U$ be a universe of discourse, $\mathscr{C}$ a family of subsets of $U$. If no subsets in $\mathscr{C}$ are empty and $\cup \mathscr{C}=U, \mathscr{C}$ is called a covering of $U$.

It is clear that a partition of $U$ is a covering of $U$; therefore the concept of a covering is an extension of the concept of a partition. In the following discussion, $\mathscr{C}$ can be an infinite covering; that is, we do not restrict the covering to the finite.

Definition 2. Let $U$ be a nonempty set, $\mathscr{C}$ a covering of $U$. The pair $(U, \mathscr{C})$ is called a covering approximation space.

Definition 3 (see [3], minimal description). Let $(U, \mathscr{C})$ be a covering approximation space and $x \in U$. The family of sets $M d_{\mathscr{C}}(x)=\{C \in \mathscr{C} \mid x \in C \wedge(\forall S \in \mathscr{C} \wedge x \in S \wedge S \subseteq C \Rightarrow$ $C=S)\}$ is called a minimal description of $x$. When there is no confusion, we omit the subscript $\mathscr{C}$.

Definition 4 (see $[3,29]$, neighborhood). Let $\mathscr{C}$ be a covering of $U ; N_{\mathscr{C}}(x)=\cap\{C \in \mathscr{C} \mid x \in C\}$ is called a neighborhood of $x$. Generally, we omit the subscript $\mathscr{C}$ when there is no confusion.

By Definition 4, it is easy to see that $\forall u \quad \epsilon$ $N_{\mathscr{C}}(x), N_{\mathscr{C}}(u) \subseteq N_{\mathscr{C}}(x)$.

In covering rough set models, seven pairs of the most commonly used approximation operators are defined as follows $[27,34]$.

Definition 5 (covering lower and upper approximation operations). Let $\mathscr{C}$ be a covering of $U$. The operations, $\mathrm{CL}_{\mathscr{C}}$ : $P(U) \rightarrow P(U)$ and $\mathrm{CL}_{\mathscr{C}}^{\prime}: P(U) \rightarrow P(U)$, are defined as follows: for all $X \in P(U)$,

$$
\begin{gathered}
\mathrm{CL}_{\mathscr{C}}(X)=\cup\{C \in \mathscr{C} \mid C \subseteq X\}, \\
\mathrm{CL}_{\mathscr{C}}^{\prime}(X)=\{x \mid N(x) \subseteq X\}=\cup\{N(x) \mid N(x) \subseteq X\} .
\end{gathered}
$$

We call $\mathrm{CL}_{\mathscr{C}}$ the first, the second, the third, the fourth, or the fifth covering lower approximation operations and
$\mathrm{CL}_{\mathscr{C}}^{\prime}$ the sixth and the seventh covering lower approximation operations with respect to the covering $\mathscr{C}$.

The operations $F H, S H, T H, R H, I H, X H, V H: P(U) \rightarrow$ $P(U)$ are defined as follows: for all $X \in P(U)$,

$$
\begin{aligned}
& F H_{\mathscr{C}}(X)=\mathrm{CL}(X) \cup(\cup\{\cup M d(x) \mid x \in X-\mathrm{CL}(X)\}), \\
& S H_{\mathscr{C}}(X)=\cup\{C \mid C \in \mathscr{C}, C \cap X \neq \emptyset\}, \\
& T H_{\mathscr{C}}(X)=\cup\{\cup M d(x) \mid x \in X\}, \\
& R H_{\mathscr{C}}(X)=\mathrm{CL}(X) \cup(\cup\{C \mid C \cap(X-\mathrm{CL}(X)) \neq \emptyset\}), \\
& I H_{\mathscr{C}}(X)=\mathrm{CL}(X) \cup(\cup\{N(x) \mid x \in X-\mathrm{CL}(X)\})= \\
& \cup\{N(x) \mid x \in X\}, \\
& X H_{\mathscr{C}}(X)=\{x \mid N(x) \cap X \neq \emptyset\}, \\
& V H_{\mathscr{C}}(X)=\cup\{N(x) \mid N(x) \cap X \neq \emptyset\} .
\end{aligned}
$$

$F H_{\mathscr{C}}, S H_{\mathscr{C}}, T H_{\mathscr{C}}, R H_{\mathscr{C}}, I H_{\mathscr{C}}, X H_{\mathscr{C}}, V H_{\mathscr{C}}$ are called the first, the second, the third, the fourth, the fifth, the sixth, and the seventh covering upper approximation operations with respect to $\mathscr{C}$, respectively. We leave out $\mathscr{C}$ at the subscript when there is no confusion.

Note 1. Since for all $C \in \mathscr{C}, C=\cup\{N(x) \mid x \in C\}$, it is easy to check that $\mathrm{CL}(X) \cup(\cup\{N(x) \mid x \in X-\mathrm{CL}(X)\})=\cup\{N(x) \mid$ $x \in X\}$ when $\mathscr{C}$ is a finite (or infinite) covering. Therefore $I H_{\mathscr{C}}(X)=\mathrm{CL}(X) \cup(\cup\{N(x) \mid x \in X-\mathrm{CL}(X)\})=\cup\{N(x) \mid$ $x \in X\}$ is still held when we do not restrict the covering to the finite.

Definition 6 (see [35] unary covering). Let $\mathscr{C}$ be a covering of a set $U . \mathscr{C}$ is called unary if for all $x \in U,|M d(x)|=1$.

\section{Relationships between $F H$ and $S H, T H, R H$, $I H, X H$, and $V H$}

In this section, we study the relationships between the first type of covering rough sets and others and present some conditions under which two types of upper approximation operations are identical.

When the covering is finite, Zhu and Wang presented the condition, " $\mathscr{C}$ is a partition," under which $F H=S H$ [35]. In the following, we show that the result is held when $\mathscr{C}$ is not restricted to the finite.

Theorem 7. Let $(U, \mathscr{C})$ be a covering approximation space. Then $\mathrm{FH}=\mathrm{SH}$ if and only if $\mathscr{C}$ is a partition.

Proof. We only need to prove the necessity. Suppose that $\mathscr{C}$ is not a partition. Then there exist $C_{1}, C_{2} \in \mathscr{C}$ such that $C_{1} \cap C_{2} \neq \emptyset$ and $C_{1} \neq C_{2}$. Hence $C_{1} \nsubseteq C_{2}$ or $C_{1} \nsupseteq C_{2}$. We may assume that $C_{1} \nsubseteq C_{2}$. By Definition 5 , we conclude that $F H\left(C_{2}\right)=C_{2}$ and $C_{1} \subseteq S H\left(C_{2}\right)$, thus $F H\left(C_{2}\right) \neq S H\left(C_{2}\right)$. This is a contradiction with the condition $\mathrm{FH}=\mathrm{SH}$. Hence $\mathscr{C}$ is a partition.

When the covering, $\mathscr{C}$, is finite, Zhu and Wang gave a condition under which $F H=T H$ [35]. His result is that "FH = TH if and only if $\mathscr{C}$ is unary." However, when the covering, $\mathscr{C}$, is infinite, the above result is not always true. In fact, Example 10 illustrates that " $F H=T H \nRightarrow \mathscr{C}$ is 
unary," and the following example illustrates that " $\mathscr{C}$ is unary $\nRightarrow F H=T H$."

Example 8. Let $U=\left\{x_{0}, x_{1}, y_{1}, x_{2}, y_{2}, x_{3}, y_{3}, \ldots\right\}, C_{0}=$ $\left\{x_{0}, x_{1}, x_{2}, \ldots\right\}, C_{i}=\left\{x_{0}, y_{i}, y_{i+1}, y_{i+2}, \ldots\right\}, i=1,2,3, \ldots$, and $\mathscr{C}=\left\{C_{i} \mid i=0,1,2, \ldots\right\}$. Clearly, $\mathscr{C}$ is a covering of $U$. It is easy to check that $M d\left(x_{i}\right)=\left\{C_{0}\right\}, i=0,1,2, \ldots$, and $\operatorname{Md}\left(y_{k}\right)=\left\{C_{k}\right\}, k=1,2,3, \ldots$. Hence $\mathscr{C}$ is unary. It is easy to see that $F H\left(C_{1}\right)=C_{1}$ and $T H\left(C_{1}\right)=U$, which implies $F H\left(C_{1}\right) \neq T H\left(C_{1}\right)$. Therefore, $F H \neq T H$.

In the following, we give a condition under which $F H=$ $T H$ when $\mathscr{C}$ is a general covering.

Theorem 9. Let $(U, \mathscr{C})$ be a covering approximation space. Then $\mathrm{FH}=\mathrm{TH}$ if and only if for all $\mathrm{C} \in \mathscr{C}, C=\cup\{\cup M d(x) \mid$ $x \in C\}$.

Proof. We first prove the necessity. Let $C \in \mathscr{C}$. Then it follows by $F H=T H$ that $F H(C)=T H(C)$. By the definitions of $F H$ and $T H, C=\cup\{\cup M d(x) \mid x \in C\}$.

Conversely, let $X \subseteq U$. By the definition of CL, there exists $\mathscr{B} \subseteq \mathscr{C}$ such that $\mathrm{CL}(X)=\cup_{C \in \mathscr{B}} C$. By the condition $\forall C \in \mathscr{C}$, $C=\cup\{\cup M d(x) \mid x \in C\}$, we have that

$$
\begin{aligned}
\mathrm{CL}(X) & =\cup_{C \in \mathscr{B}} C=\cup_{C \in \mathscr{B}}(\cup\{\cup M d(x) \mid x \in C\}) \\
& =\cup\{\cup M d(x) \mid x \in C, C \in \mathscr{B}\} \\
& =\cup\left\{\cup M d(x) \mid x \in \cup_{C \in \mathscr{B}} C\right\} \\
& =\cup\{\cup M d(x) \mid x \in \mathrm{CL}(X)\}
\end{aligned}
$$

that is, $\mathrm{CL}(X)=\cup\{\cup M d(x) \mid x \in \mathrm{CL}(X)\}$. Hence

$$
\begin{aligned}
F H(X)= & C L(X) \cup(\cup\{\cup M d(x) \mid x \in X-\mathrm{CL}(X)\}) \\
= & (\cup\{\cup M d(x) \mid x \in \mathrm{CL}(X)\}) \\
& \cup(\cup\{\cup M d(x) \mid x \in X-\mathrm{CL}(X)\}) \\
= & \cup\{\cup M d(x) \mid x \in X\}=T H(X) .
\end{aligned}
$$

We have proved that, $\forall X \subseteq U, F H(X)=T H(X)$. Therefore, $F H=T H$.

Applying the above result, we can give the following example which illustrates that " $F H=T H \nRightarrow \mathscr{C}$ is unary."

Example 10. Let $U=\left\{x_{0}, x_{1}, x_{2}, \ldots\right\}, C_{i}=\left\{x_{0}, x_{i}, x_{i+1}\right.$, $\left.x_{i+2}, \ldots\right\}, i=1,2, \ldots$, and $\mathscr{C}=\left\{C_{i} \mid i=1,2, \ldots\right\}$. Clearly, $\mathscr{C}$ is a covering. It is clear that $M d\left(x_{i}\right)=\left\{C_{i}\right\}, i=1,2, \ldots$ Hence $C_{i}=\cup\left\{\cup M d(x) \mid x \in C_{i}\right\}, i=1,2, \ldots$. It follows from Theorem 9 that $F H=T H$. However, we can prove that $M d\left(x_{0}\right)=\emptyset$; that is, $\left|M d\left(x_{0}\right)\right|=0$, and thus $\mathscr{C}$ is not unary. Suppose that $\left|M d\left(x_{0}\right)\right| \neq 0$; that is, $M d\left(x_{0}\right) \neq \emptyset$; then there exists $C_{k} \in M d\left(x_{0}\right)$. However, by the definition of $\mathscr{C}$, we have that $x_{0} \in C_{k+1}$ and $C_{k+1} \varsubsetneqq C_{k}$, which is a contradiction with $C_{k} \in M d(x)$. Hence $\left|M d\left(x_{0}\right)\right|=0$.

When the covering, $\mathscr{C}$, is finite, Zhu and Wang obtained a condition under which $F H=R H$ [30]. The result is still true when $\mathscr{C}$ is a general covering and the proof is similar.
Theorem 11 (see [30]). Let $(U, \mathscr{C})$ be a covering approximation space. Then $F H=R H$ if and only if $\mathscr{C}$ satisfies the following condition: for all $x \in U$, if $\{x\} \notin \mathscr{C}$, then, $\forall C \in \mathscr{C}$ and $x \in C, C \subseteq M d(x)$.

There are no studies on the relationships $\mathrm{FH}$ and $\mathrm{IH}, \mathrm{XH}$, and $V H$. In the following, we discuss these problems. First, we establish a condition under which $F H$ and $I H$ are identical.

Theorem 12. Let $(U, \mathscr{C})$ be a covering approximation space. Then $F H=I H$ if and only if, for all $x \in U, N(x)=U M d(x)$.

Proof. The sufficiency follows directly from the definitions of $\mathrm{FH}$ and $\mathrm{IH}$.

Conversely, suppose that there exists $x \in U$ such that $N(x) \neq \cup M d(x)$. We will show that $\{x\} \notin \mathscr{C}$. Otherwise, $\{x\} \in \mathscr{C}$; then we can get that $N(x)=\{x\}=\cup M d(x)$, a contradiction. Thus $\{x\} \notin \mathscr{C}$ and so $\mathrm{CL}(\{x\})=\emptyset$. It follows from Definition 5 that $F H(\{x\})=\cup M d(x)$ and $I H(\{x\})=$ $N(x)$. Thus $F H(\{x\}) \neq I H(\{x\})$, which contradicts the condition, $F H=I H$. This completes the proof of the necessity.

It is worth noting that in Example $8, \mathscr{C}$ is unary; however, $N\left(x_{0}\right)=\left\{x_{0}\right\} \neq C_{0}=M d\left(x_{0}\right)$. That is, $\mathscr{C}$ is unary $\nRightarrow \forall x \in U$, $N(x)=\cup M d(x)$.

Lemma 13. Let $(U, \mathscr{C})$ be a covering approximation space and $X \subseteq U$. If, $\forall C \in \mathscr{C}, \forall u \in U \backslash C, N(u) \cap C=\emptyset$, then $C L(X)=$ $\cup\{N(x) \mid N(x) \cap C L(X) \neq \emptyset\}=\{x \mid N(x) \cap C L(X) \neq \emptyset\}$.

Proof. (i) We will show that, $\forall C \in \mathscr{C}, C=\cup\{N(x) \mid N(x) \cap$ $C \neq \emptyset$. Let $C \in \mathscr{C}$. For any $y \in C$, clearly, $y \in N(y) \cap C \neq \emptyset$, and thus $y \in \cup\{N(x) \mid N(x) \cap C \neq \emptyset\}$ and so $C \subseteq \cup\{N(x) \mid$ $N(x) \cap C \neq \emptyset\}$. On the other hand, for all $y \in \cup\{N(x) \mid N(x) \cap$ $C \neq \emptyset\}$, there exists $x \in U$ such that $N(x) \cap C \neq \emptyset$ and $y \in$ $N(x)$. Suppose $x \in U \backslash C$; then it follows from the assumed condition that $N(x) \cap C=\emptyset$, which is a contradiction. Hence $x \in C$ and so $N(x) \subseteq C$, which implies that $y \in C$. Thus, $\cup\{N(x) \mid N(x) \cap C \neq \emptyset\} \subseteq C$. In summary, $C=\cup\{N(x) \mid$ $N(x) \cap C \neq \emptyset\}$.

(ii) By the definition of CL, there exists $\mathscr{B} \subseteq \mathscr{C}$ such that $\mathrm{CL}(X)=\cup_{C \in \mathscr{B}} C$. By (i), we have that

$$
\begin{aligned}
\mathrm{CL}(X) & =\cup_{C \in \mathscr{B}} C=\cup_{C \in \mathscr{B}}(\cup\{N(x) \mid N(x) \cap C \neq \emptyset\}) \\
& =\cup\{N(x) \mid N(x) \cap C \neq \emptyset, C \in \mathscr{B}\} \\
& =\cup\left\{N(x) \mid N(x) \cap\left(\cup_{C \in \mathscr{B}} C\right) \neq \emptyset\right\} \\
& =\cup\{N(x) \mid N(x) \cap \mathrm{CL}(X) \neq \emptyset\}
\end{aligned}
$$

that is, $\mathrm{CL}(X)=\cup\{N(x) \mid N(x) \cap \mathrm{CL}(X) \neq \emptyset\}$.

(iii) We will show that $\forall C \in \mathscr{C}, C=\{x \mid N(x) \cap C \neq \emptyset\}$. By Definition 4, it is clear that $C \subseteq\{x \mid N(x) \cap C \neq \emptyset\}$. Clearly, $\{x \mid N(x) \cap C \neq \emptyset\} \subseteq \cup\{N(x) \mid N(x) \cap C \neq \emptyset\}$; thus by (i), $\{x \mid N(x) \cap C \neq \emptyset\} \subseteq C$. Therefore, $C=\{x \mid N(x) \cap C \neq \emptyset\}$.

(iv) By (iii), the proof of $\mathrm{CL}(X)=\{x \mid N(x) \cap \mathrm{CL}(X) \neq \emptyset\}$ is similar to that of (ii).

Thus, by (ii) and (iv), $\mathrm{CL}(X)=\cup\{N(x) \mid N(x) \cap \mathrm{CL}(X) \neq$ $\emptyset\}=\{x \mid N(x) \cap \mathrm{CL}(X) \neq \emptyset\}$. 
Lemma 14. Let $(U, \mathscr{C})$ be a covering approximation space. If, $\forall x \in U, \cup M d(x)=\{u \mid x \in N(u)\}$, then, $\forall Y \subseteq U$, $\cup\{\cup M d(x) \mid x \in Y\}=\{x \mid N(x) \cap Y \neq \emptyset\}$.

Proof. Let $Y \subseteq U$. Since, $\forall x \in U, \cup M d(x)=\{u \mid x \in N(u)\}$, it follows that

$$
\begin{aligned}
y \in \cup\{\cup M d(x) \mid x \in Y\} & \Longleftrightarrow \exists x \in Y, \quad y \in \cup M d(x) \\
& \Longleftrightarrow \exists x \in Y, \quad y \in\{u \mid x \in N(u)\} \\
& \Longleftrightarrow \exists x \in Y, \quad x \in N(y) \\
& \Longleftrightarrow N(y) \cap Y \neq \emptyset \\
& \Longleftrightarrow y \in\{x \mid N(x) \cap Y \neq \emptyset\} .
\end{aligned}
$$

Therefore, $\cup\{\cup M d(x) \mid x \in Y\}=\{x \mid N(x) \cap Y \neq \emptyset\}$.

In the following, we give a condition under which $\mathrm{FH}$ and $\mathrm{XH}$ are identical.

Theorem 15. Let $(U, \mathscr{C})$ be a covering approximation space. Then $F H=X H$ if and only if, $\forall C \in \mathscr{C}, \forall u \in U \backslash C, N(u) \cap C=$ $\emptyset$ and $\forall x \in U, \cup M d(x)=\{u \mid x \in N(u)\}$.

Proof. We first prove the necessity.

(i) We will show that, $\forall C \in \mathscr{C}, \forall u \in U \backslash C, N(u) \cap C=\emptyset$. Suppose that, $\exists C \in \mathscr{C}, \exists u \in U \backslash C, N(u) \cap C \neq \emptyset$. Then by Definition 5, we have that $F H(C)=C$ and $X H(C)=\{x \mid$ $N(x) \cap C \neq \emptyset\}$; thus by $N(u) \cap C \neq \emptyset, u \in X H(C)$. Since $u \in U \backslash C$, it follows that $u \notin C$ and so $u \notin F H(C)$. Hence $F H(C) \neq X H(C)$, which is a contradiction with the assumed condition $F H=X H$. Thus, $\forall C \in \mathscr{C}, \forall u \in U \backslash C, N(u) \cap C=\emptyset$.

(ii) We will show that, $\forall x \in U, \cup M d(x)=\{u \mid x \in N(u)\}$. Let $x \in U$. If $\{x\} \in \mathscr{C}$, then $\mathrm{CL}(\{x\})=\{x\}$ and by Definition 3 , we have that $\operatorname{Md}(x)=\{\{x\}\}$. Hence $F H(\{x\})=\{x\}$ and $\cup M d(x)=\{x\}$. Therefore, by $F H=X H$, we can get that

$$
\begin{aligned}
\cup M d(x) & =F H(\{x\})=X H(\{x\})=\{u \mid N(u) \cap\{x\} \neq \emptyset\} \\
& =\{u \mid x \in N(u)\}
\end{aligned}
$$

that is, $\cup M d(x)=\{u \mid x \in N(u)\}$. If $\{x\} \notin \mathscr{C}$, then $\operatorname{CL}(\{x\})=$ $\emptyset$ and so $F H(\{x\})=U M d(x)$. In addition, $X H(\{x\})=\{u \mid$ $N(u) \cap\{x\} \neq \emptyset\}=\{u \mid x \in N(u)\}$. By $F H=X H$, we conclude that $F H(\{x\})=X H(\{x\})$ and so $\cup M d(x)=\{u \mid x \in N(u)\}$.

By (i) and (ii), the necessity holds.

Conversely, let $X \subseteq U$. By Lemma 13, we have that $\mathrm{CL}(X)=\{x \mid N(x) \cap \mathrm{CL}(X) \neq \emptyset\}$. By Lemma 14, we have that $\cup\{\mathrm{UMd}(x) \mid x \in X-\mathrm{CL}(X)\}=\{x \mid N(x) \cap(X-\mathrm{CL}(X)) \neq \emptyset\}$. Thus

$$
\begin{aligned}
F H(X)= & C L(X) \cup(\cup\{\cup M d(x) \mid x \in X-\operatorname{CL}(X)\}) \\
= & \{x \mid N(x) \cap \mathrm{CL}(X) \neq \emptyset\} \\
& \cup\{x \mid N(x) \cap(X-\operatorname{CL}(X)) \neq \emptyset\} \\
= & \{x \mid N(x) \cap X \neq \emptyset\}=X H(X) .
\end{aligned}
$$

We have proved that, $\forall X \subseteq U, F H(X)=X H(X)$. Therefore, $F H=X H$.
Lemma 16. Let $(U, \mathscr{C})$ be a covering approximation space. If, $\forall x \in U, \cup M d(x)=\cup\{N(u) \mid x \in N(u)\}$, then, $\forall Y \subseteq U$, $\cup\{\cup M d(x) \mid x \in Y\}=\cup\{N(x) \mid N(x) \cap Y \neq \emptyset\}$.

Proof. Let $Y \subseteq U$. Since, $\forall x \in U, \cup M d(x)=\cup\{N(u) \mid x \in$ $N(u)$, it follows that

$$
\begin{aligned}
y & \in \cup\{\cup M d(x) \mid x \in Y\} \\
& \Longleftrightarrow \exists x \in Y, \quad y \in \cup M d(x) \\
& \Longleftrightarrow \exists x \in Y, \quad y \in \cup\{N(u) \mid x \in N(u)\} \\
& \Longleftrightarrow \exists x \in Y, \quad \exists u \in U, \quad x \in N(u), \quad y \in N(u) \\
& \Longleftrightarrow \exists u \in U, \quad N(u) \cap Y \neq \emptyset, \quad y \in N(u) \\
& \Longleftrightarrow y \in \cup\{N(x) \mid N(x) \cap Y \neq \emptyset\} .
\end{aligned}
$$

Therefore, $\cup\{\cup M d(x) \mid x \in Y\}=\cup\{N(x) \mid N(x) \cap Y \neq$ $\emptyset\}$.

In the following, we present a condition under which $F H$ and $\mathrm{VH}$ are identical.

Theorem 17. Let $(U, \mathscr{C})$ be a covering approximation space. Then $F H=V H$ if and only if, $\forall C \in \mathscr{C}, \forall u \in U \backslash C, N(u) \cap C=$ $\emptyset$ and $\forall x \in U, \cup M d(x)=\cup\{N(u) \mid x \in N(u)\}$.

Proof. We first prove the necessity.

(i) We will show that, $\forall C \in \mathscr{C}, \forall u \in U \backslash C, N(u) \cap C=\emptyset$. Suppose that, $\exists C \in \mathscr{C}, \exists u \in U \backslash C, N(u) \cap C \neq \emptyset$. Then by Definition 5, we have that $F H(C)=C$ and $V H(C)=\cup\{N(x) \mid$ $N(x) \cap C \neq \emptyset\}$; thus by $N(u) \cap C \neq \emptyset, u \in V H(C)$. Since $u \in$ $U \backslash C$, it follows that $u \notin C$ and so $u \notin F H(C)$. Hence $F H(C) \neq$ $V H(C)$, which is a contradiction with the condition, $F H=$ $V H$. Thus, $\forall C \in \mathscr{C}, \forall u \in U \backslash C, N(u) \cap C=\emptyset$.

(ii) We will show that, $\forall x \in U, \cup M d(x)=\{N(u) \mid x \in$ $N(u)\}$. Let $x \in U$. If $\{x\} \in \mathscr{C}$, then $\operatorname{CL}(\{x\})=\{x\}$ and by Definition 3, we have that $M d(x)=\{\{x\}\}$. Hence $F H(\{x\})=$ $\{x\}$ and $\cup M d(x)=\{x\}$. By $F H=V H$, we have that $\cup M d(x)=$ $F H(\{x\})=V H(\{x\})=\bigcup\{N(u) \mid N(u) \cap\{x\} \neq \emptyset\}=\bigcup\{N(u) \mid$ $x \in N(u)\}$. Thus $\cup M d(x)=\cup\{N(u) \mid x \in N(u)\}$. If $\{x\} \notin \mathscr{C}$, then $\operatorname{CL}(\{x\})=\emptyset$ and so $F H(\{x\})=\cup M d(x)$. In addition, $V H(\{x\})=\cup\{N(u) \mid N(u) \cap\{x\} \neq \emptyset\}=\cup\{N(u) \mid x \in N(u)\}$. By $F H=V H$, we conclude that $F H(\{x\})=V H(\{x\})$ and so $\cup M d(x)=\cup\{N(u) \mid x \in N(u)\}$.

(iii) By (i) and (ii), the necessity holds.

Conversely, let $X \subseteq U$. By Lemma 13, we have that $\mathrm{CL}(X)=\cup\{N(x) \mid N(x) \cap \mathrm{CL}(X) \neq \emptyset\}$. By Lemma 16, $\cup\{\cup M d(x) \mid x \in X-\mathrm{CL}(X)\}=\cup\{N(x) \mid N(x) \cap(X-$ $\mathrm{CL}(X)) \neq \emptyset\}$. Thus

$$
\begin{aligned}
F H(X)= & C L(X) \cup(\cup\{\cup M d(x) \mid x \in X-\mathrm{CL}(X)\}) \\
= & \cup\{N(x) \mid N(x) \cap \mathrm{CL}(X) \neq \emptyset\} \\
& \cup(\cup\{N(x) \mid N(x) \cap(X-\mathrm{CL}(X)) \neq \emptyset\}) \\
= & \cup\{N(x) \mid N(x) \cap X \neq \emptyset\}=V H(X) .
\end{aligned}
$$

We have proved that, $\forall X \subseteq U, F H(X)=V H(X)$. Therefore, $F H=V H$. 


\section{Relationships between $S H$ and $T H, R H, I H$, $X H$, and $V H$}

In this section, we explore the relationships between the second type of covering rough sets and later five types, and establish the conditions under which two types of upper approximation operations are identical.

When the covering, $\mathscr{C}$, is finite, Zhu and Wang gave a condition under which $\mathrm{SH}$ and $\mathrm{TH}$ are identical in [35]. In the following part, we show that their conclusion is still held when the covering $\mathscr{C}$ is a general covering.

Theorem 18. Let $(U, \mathscr{C})$ be a covering approximation space. Then $S H=T H$ if and only if, $\forall C \in \mathscr{C}, \forall x \in C, C \subseteq \cup M d(x)$.

Proof. Let $C \in \mathscr{C}$ and $x \in C$; then $C \cap\{x\} \neq \emptyset$. By Definition 5, we conclude that $C \subseteq S H(\{x\})$ and $T H(\{x\})=\cup M d(x)$. By $S H=T H, S H(\{x\})=T H(\{x\})$ and so $C \subseteq \cup M d(x)$. This finishes the proof of necessity.

Conversely, let $X \subseteq U$. By Definitions 3 and 5, it is easy to see that $T H(X) \subseteq S H(X)$. On the other hand, $\forall y \in S H(X)$, then, $\exists C \in \mathscr{C}, C \cap X \neq \emptyset$ and $y \in C$. Taking $x \in C \cap X$, then $x \in C$ and $x \in X$. Thus $\cup M d(x) \subseteq T H(X)$. By our condition, we have that $C \subseteq \cup M d(x)$. Hence $y \in T H(X)$. Thus $S H(X) \subseteq$ $T H(X)$. In summary, $T H(X)=S H(X)$. We have proved that, $\forall X \subseteq U, T H(X)=S H(X)$. Therefore, $S H=T H$.

Now we present some conditions under which $S H=R H$, $S H=I H$, and $S H=X H$.

Theorem 19. Let $(U, \mathscr{C})$ be a covering approximation space. Then the following conditions are equivalent.

(1) $S H=R H$.

(2) $S H=I H$.

(3) $\mathrm{SH}=X H$.

(4) $\mathscr{C}$ is a partition.

Proof. (1) $\Rightarrow$ (4) Suppose that $\mathscr{C}$ is not a partition; then there exist $C_{1}, C_{2} \in \mathscr{C}$ such that $C_{1} \cap C_{2} \neq \emptyset$ and $C_{1} \neq C_{2}$. Hence $C_{1} \nsubseteq C_{2}$ or $C_{1} \nsupseteq C_{2}$. We may assume that $C_{1} \nsubseteq C_{2}$. By Definition 5, we have that $R H\left(C_{2}\right)=C_{2}$ and $C_{1} \subseteq S H\left(C_{2}\right)$; thus $\mathrm{RH}\left(\mathrm{C}_{2}\right) \neq \mathrm{SH}\left(\mathrm{C}_{2}\right)$. This is a contradiction with the condition, $S H=R H$. Hence $\mathscr{C}$ is a partition.

$(2) \Rightarrow(4)$ The proof is similar to that of " $(1) \Rightarrow(4)$ ".

(3) $\Rightarrow$ (4) Suppose that $\mathscr{C}$ is not a partition; then there exist $C_{1}, C_{2} \in \mathscr{C}$ such that $C_{1} \neq C_{2}$ and $C_{1} \cap C_{2} \neq \emptyset$. Thus $C_{1} \nsubseteq C_{2}$ or $C_{2} \nsubseteq C_{1}$. Without loss of generality, we assume that $C_{1} \nsubseteq C_{2}$. Taking $y \in C_{1}-C_{2}$ and $z \in C_{1} \cap C_{2}$, by Definition 4 , we have that $N(z) \subseteq C_{1} \cap C_{2}$. Since $y \notin C_{2}$, it follows that $y \notin N(z)$; that is, $N(z) \cap\{y\}=\emptyset$. Hence by the definition of $X H$, we have that $z \notin X H(\{y\})$. On the other hand, by $y \in C_{1} \cap\{y\} \neq \emptyset$ and the definition of $S H$, we have that $C_{1} \subseteq S H(\{y\})$. Since $z \in C_{1} \cap C_{2}$, it follows that $z \in$ $S H(\{y\})$. Thus $S H(\{y\}) \neq X H(\{y\})$, which is a contradiction with the condition $S H=X H$. This completes the proof.

$(4) \Rightarrow(1),(2),(3)$ It follows directly from Definition 5.
By Theorems 7 and 19, we can conclude that $S H=F H \Leftrightarrow$ $S H=R H \Leftrightarrow S H=I H \Leftrightarrow S H=X H \Rightarrow F H=R H=I H=$ $X H$.

In the following, we give a condition under which $\mathrm{SH}$ and $\mathrm{VH}$ are identical.

Theorem 20. Let $(U, \mathscr{C})$ be a covering approximation space. Then $S H=V H$ if and only if, $\forall C \in \mathscr{C}, \forall y, z \in C$, there exists $x \in U$ such that $y, z \in N(x)$.

Proof. We first prove the necessity. $\forall C \in \mathscr{C}, \forall y, z \in C$, we will show that, $\exists x \in U, y, z \in N(x)$. Clearly, $C \cap\{z\} \neq \emptyset$, hence by the definition of $S H$ we have that $C \subseteq S H(\{z\})$ and so $y \in S H(\{z\})$. By $S H=V H$, we can get that $S H(\{z\})=$ $V H(\{z\})$, and thus $y \in V H(\{z\})$. According to the definition of $V H$, we conclude that there exists $x \in U$ such that $N(x) \cap$ $\{z\} \neq \emptyset$ and $y \in N(x)$. Since $N(x) \cap\{z\} \neq \emptyset$ implies that $z \in N(x)$, it follows that $y, z \in N(x)$. This finishes the proof of the necessity.

Conversely, let $X \subseteq U$. Then by the definitions of $S H$ and $V H$, it is easy to check that $V H(X) \subseteq S H(X)$. On the other hand, $\forall y \in S H(X)$, by the definition of $S H$, there exists $C \in \mathscr{C}$ such that $C \cap X \neq \emptyset$ and $y \in C$. Taking $z \in C \cap X$, then $y, z \in C$. By the assumed condition, there exists $x \in U$ such that $y, z \in N(x)$. Clearly, $z \in N(x) \cap X \neq \emptyset$, and thus by the definition of $V H$, we have that $N(x) \subseteq V H(X)$ and so $y \in V H(X)$. Hence $S H(X) \subseteq V H(X)$. In summary, $S H(X)=$ $V H(X)$. We have proved that, $\forall X \subseteq U, S H(X)=V H(X)$. Therefore, $\mathrm{SH}=\mathrm{VH}$.

\section{Relationships between $T H$ and $R H, I H, X H$, and $V H$}

In this section, we study the relationships between the third type of covering rough sets and later four types and establish the conditions under which two types of upper approximation operations are identical.

Lemma 21. Let $(U, \mathscr{C})$ be a covering approximation space and $X \subseteq U$. Then, $\forall x \in X-C L(X),\{x\} \notin \mathscr{C}$.

Proof. Let $x \in X-\mathrm{CL}(X)$. Then $x \in X$ and $x \notin \mathrm{CL}(X)$. If $\{x\} \in \mathscr{C}$, then by $\{x\} \subseteq X$ and the definition of CL we have that $x \in \mathrm{CL}(X)$, a contradiction. Therefore, $\{x\} \notin \mathscr{C}$.

Lemma 22. Let $(U, \mathscr{C})$ be a covering approximation space and $X \subseteq U$. If $\forall C_{1}, C_{2} \in \mathscr{C}, C_{1} \neq C_{2}$ and $x \in C_{1} \cap C_{2}$ imply that $\{x\} \in \mathscr{C}$, then the following assertions hold.

(1) $\forall C \in \mathscr{C}, C=\cup\{\cup M d(x) \mid x \in C\}$.

(2) $\cup\{\cup M d(x) \mid x \in X-C L(X)\}=\cup\{K \mid K \cap(X-$ $C L(X)) \neq \emptyset\}$

Proof. (1) Let $C \in \mathscr{C}$. For all $y \in C$, if $C \in M d(y)$, then $y \in \cup\{\cup M d(x) \mid x \in C\}$. If $C \notin M d(y)$, then, by Definition 3, there exists $K \in \mathscr{C}$ such that $y \in K$ and $K \varsubsetneqq C$. Hence $K \neq C$ and $y \in K \cap C$, which implies that $\{y\} \in \mathscr{C}$ by the assumed condition. Thus $M d(y)=\{\{y\}\}$ and so $y \in \cup\{\cup M d(x) \mid x \in$ $C\}$. It follows that $C \subseteq \cup\{\cup M d(x) \mid x \in C\}$. On the other 
hand, $\forall y \in \cup\{\cup M d(x) \mid x \in C\}$, then, $\exists x \in C, y \in \cup M d(x)$. Thus, $\exists K \in M d(x), x, y \in K$. If $K=C$, then $y \in C$. If $K \neq C$, then by, $x \in K \cap C$, it follows from the assumed condition that $\{x\} \in \mathscr{C}$. Hence $M d(x)=\{\{x\}\}$. By $K \in M d(x)$, we have that $K=\{x\}$. By $y \in K$, we have that $x=y$ and so $y \in C$. Therefore, $\cup\{\cup M d(x) \mid x \in C\} \subseteq C$. In summary, $C=\cup\{\cup M d(x) \mid x \in C\}$.

(2) $\forall y \in \cup\{\cup M d(x) \mid x \in X-\mathrm{CL}(X)\}$, then, $\exists x \in X-$ $\mathrm{CL}(X), y \in \cup M d(x)$. This implies that, $\exists C \in M d(x), y \in C$. Since $x \in C \cap(X-\mathrm{CL}(X)) \neq \emptyset$, it follows that $C \in\{C \mid$ $C \cap(X-\mathrm{CL}(X)) \neq \emptyset\}$ and so $y \in \cup\{C \mid C \cap(X-\mathrm{CL}(X)) \neq \emptyset\}$. Therefore, $\cup\{\cup M d(x) \mid x \in X-\mathrm{CL}(X)\} \subseteq \cup\{C \mid C \cap(X-$ $\mathrm{CL}(X)) \neq \emptyset\}$. On the other hand, $\forall y \in \cup\{C \mid C \cap(X-$ $\mathrm{CL}(X)) \neq \emptyset\}$, then there exists $C \in \mathscr{C}$ such that $C \cap(X-$ $\mathrm{CL}(X)) \neq \emptyset$ and $y \in C$. Taking $z \in C \cap(X-\mathrm{CL}(X))$, then $z \in$ $X-\mathrm{CL}(X)$ and by Lemma 21 , we have that $\{z\} \notin \mathscr{C}$. Suppose that $C \notin M d(z)$, then by Definition 3, there exists $K \in \mathscr{C}$ such that $z \in K$ and $K \varsubsetneqq C$. Thus $z \in K \cap C$, by our condition, this implies that $\{z\} \in \mathscr{C}$, a contradiction with $\{z\} \notin \mathscr{C}$. Hence $C \in M d(z)$ and so by $z \in X-\mathrm{CL}(X), C \subseteq \cup\{\cup M d(x) \mid x \in$ $X-\mathrm{CL}(X)\}$. It follows that $y \in \cup\{\cup M d(x) \mid x \in X-\mathrm{CL}(X)\}$. Thus $\cup\{C \mid C \cap(X-\mathrm{CL}(X)) \neq \emptyset\} \subseteq \cup\{\cup M d(x) \mid x \in$ $X-\mathrm{CL}(X)\}$. In summary, $\cup\{\cup M d(x) \mid x \in X-\mathrm{CL}(X)\}=$ $\cup\{C \mid C \cap(X-\operatorname{CL}(X)) \neq \emptyset\}$.

When a covering is finite, Zhu and Wang presented a condition under which $T H=R H$ [30]. In the following, we show that the result is held when we do not restrict $\mathscr{C}$ to the finite.

Theorem 23. Let $(U, \mathscr{C})$ be a covering approximation space. Then $T H=R H$ if and only if for $C_{1}, C_{2} \in \mathscr{C}, C_{1} \neq C_{2}$ and $x \in C_{1} \cap C_{2},\{x\} \in \mathscr{C}$.

Proof. Suppose that, $\exists C_{1}, C_{2} \in \mathscr{C}, C_{1} \neq C_{2}$, and $\exists y \in C_{1} \cap C_{2}$, $\{y\} \notin \mathscr{C}$. Then $\mathrm{CL}(\{y\})=\emptyset$. By the definitions of $T H$ and $R H$, we have that $T H(\{y\})=\cup M d(y)$ and $C_{1}, C_{2} \subseteq R H(\{y\})$. On the other hand, by $T H=R H$, we have that $T H\left(C_{1}\right)=$ $R H\left(C_{1}\right)$ and $T H\left(C_{2}\right)=R H\left(C_{2}\right)$. Thus $\cup\left\{\cup M d(x) \mid x \in C_{1}\right\}=$ $C_{1}$ and $\cup\left\{\cup M d(x) \mid x \in C_{2}\right\}=C_{2}$. Hence by $y \in C_{1}$ and $y \in C_{2}$, we can get that $\cup M d(y) \subseteq C_{1}$ and $\cup M d(y) \subseteq C_{2}$. This implies that $\cup M d(y) \subseteq C_{1} \cap C_{2}$ and so $T H(\{y\}) \subseteq C_{1} \cap C_{2}$. It follows by $C_{1} \neq C_{2}$ that $T H(\{y\}) \subsetneq C_{1} \cup C_{2} \subseteq R H(\{y\})$; that is $T H(\{y\}) \neq R H(\{y\})$, which is a contradiction with the condition $T H=R H$. This finishes the proof of necessity.

Conversely, let $X \subseteq U$. By the definition of CL, we have that, $\exists \mathscr{B} \subseteq \mathscr{C}, \mathrm{CL}(X)=\cup_{C \in \mathscr{B}} C$. By the part (1) of Lemma 22, we know that, $\forall C \in \mathscr{C}, C=\cup\{\cup M d(x) \mid x \in C\}$. Thus,

$$
\begin{aligned}
\mathrm{CL}(X) & =\cup_{C \in \mathscr{B}} C=\cup_{C \in \mathscr{B}}(\cup\{\cup M d(x) \mid x \in C\}) \\
& =\cup\{\cup M d(x) \mid x \in C, C \in \mathscr{B}\} \\
& =\cup\left\{\cup M d(x) \mid x \in \cup_{C \in \mathscr{B}} C\right\} \\
& =\cup\{\cup M d(x) \mid x \in \operatorname{CL}(X)\} ;
\end{aligned}
$$

that is, $\mathrm{CL}(X)=\cup\{\cup M d(x) \mid x \in \mathrm{CL}(X)\}$. Further, by the part (2) of Lemma 22, we know that $\cup\{\cup M d(x) \mid x \in X-$ $\mathrm{CL}(X)\}=\cup\{K \mid K \cap(X-\mathrm{CL}(X)) \neq \emptyset\}$. Therefore,

$$
\begin{aligned}
R H(X)= & \mathrm{CL}(X) \cup(\cup\{K \mid K \cap(X-\mathrm{CL}(X)) \neq \emptyset\}) \\
= & \cup\{\cup M d(x) \mid x \in \mathrm{CL}(X)\} \\
& \cup(\cup\{\cup M d(x) \mid x \in X-\mathrm{CL}(X)\}) \\
= & \cup\{\cup M d(x) \mid x \in X\}=T H(X) ;
\end{aligned}
$$

that is, $R H(X)=T H(X)$. We have proved that, $\forall X \subseteq U$, $R H(X)=T H(X)$. Therefore, $R H=T H$.

Now we provide a condition under which $T H$ and $I H$ are identical.

Theorem 24. Let $(U, \mathscr{C})$ be a covering approximation space. Then $T H=I H$ if and only if, $\forall x \in U, N(x)=U M d(x)$.

Proof. The sufficiency follows directly from the definitions of $T H$ and $I H$.

Conversely, suppose that there exists $x \in U$ such that $N(x) \neq \cup M d(x)$. Then by Definition 5 , we have that $T H(\{x\})=\cup M d(x)$ and $I H(\{x\})=N(x)$. Thus $T H(\{x\}) \neq$ $I H(\{x\})$, which contradicts the condition $F H=I H$. This finishes the proof of the necessity.

By Theorems 12 and 24, we can conclude that $F H=I H \Leftrightarrow$ $T H=I H \Rightarrow F H=T H$.

In the following, we give a condition of necessity under which $T H$ and $I H$ are identical.

Proposition 25. Let $(U, \mathscr{C})$ be a covering approximation space. If $T H=I H$, then, $\forall C \in \mathscr{C}, C=\cup\{\cup M d(x) \mid x \in C\}$.

Proof. Let $C \in \mathscr{C}$; then by Definition 5, we have that $T H(C)=$ $\cup\{\cup M d(x) \mid x \in C\}$ and $I H(C)=C$. Since $T H=I H$, it follows that $\mathrm{C}=\cup\{\cup M d(x) \mid x \in C\}$.

The following example shows that the converse of the above result is not true.

Example 26. Let $U=\left\{x_{0}, x_{1}, x_{2}, x_{3}, \ldots\right\}, C_{i}=$ $\left\{x_{0}, x_{i}, x_{i+1}, x_{i+2}, \ldots\right\}, i=1,2,3, \ldots$, and $\mathscr{C}=\left\{C_{i} \mid\right.$ $i=1,2,3, \ldots\}$. Clearly, $\mathscr{C}$ is a covering. By Example 10, $M d\left(x_{0}\right)=\emptyset$ and $M d\left(x_{i}\right)=\left\{C_{i}\right\}, i=1,2,3, \ldots .$. It is easy to see that, $\forall C_{i} \in \mathscr{C}, C_{i}=\cup\left\{\cup M d(x) \mid x \in C_{i}\right\}$. However, by $N\left(x_{0}\right)=\left\{x_{0}\right\} \neq \emptyset=\cup M d\left(x_{0}\right)$, it follows from Theorem 24 that $T H \neq I H$.

However, when the covering $\mathscr{C}$ is finite, the converse of Proposition 25 is true.

Theorem 27. Let $(U, \mathscr{C})$ be a covering approximation space. If $\mathscr{C}$ is a finite covering, then the following conditions are equivalent.

(1) $\mathrm{TH}=I H$.

(2) $\forall C \in \mathscr{C}, C=\cup\{\cup M d(x) \mid x \in C\}$.

(3) $\forall x \in U, N(x)=\cup M d(x)$. 
Proof. (1) $\Rightarrow$ (2) It comes from Proposition 25.

$(2) \Rightarrow$ (3) Suppose that, $\exists x_{0} \in U, N\left(x_{0}\right) \neq \cup M d\left(x_{0}\right)$. Since $\mathscr{C}$ is a finite covering, it follows from Definition 3 that $M d\left(x_{0}\right) \neq \emptyset$. Taking $K \in M d\left(x_{0}\right)$, we will show that, $\exists C \in \mathscr{C}$ and $x_{0} \in C, K \nsubseteq C$. Otherwise, $\forall C \in \mathscr{C}$ and $x_{0} \in C, K \subseteq C$, by Definitions 3 and 4, which implies that $N\left(x_{0}\right)=K=\cup M d\left(x_{0}\right)$. This contradicts the assumption $N\left(x_{0}\right) \neq \cup M d\left(x_{0}\right)$. Hence there exists $C \in \mathscr{C}$ and $x_{0} \in C$ such that $K \nsubseteq C$. $x_{0} \in C$ and $K \in M d\left(x_{0}\right)$ imply that $K \subseteq$ $\cup\{\cup M d(x) \mid x \in C\}$ and so $C \neq \cup\{\cup M d(x) \mid x \in C\}$, which is a contradiction with the condition (2). This completes the proof.

(3) $\Rightarrow$ (1) It comes from Theorem 24 .

When the covering is finite, by Theorems 9, 12, and 27, we can conclude that $F H=I H \Leftrightarrow T H=I H \Leftrightarrow F H=T H$.

In the following, we present a condition under which $\mathrm{TH}$ and $X H$ are identical.

Theorem 28. Let $(U, \mathscr{C})$ be a covering approximation space. Then $T H=X H$ if and only if, $\forall x \in U, \cup M d(x)=\{u \mid x \in$ $N(u)\}$.

Proof. We first prove the necessity. $\forall x \in U$, by Definition 5, we have that $T H(\{x\})=\cup M d(x)$ and $X H(\{x\})=\{u \mid N(u) \cap$ $\{x\} \neq \emptyset\}=\{u \mid x \in N(u)\}$. By the condition $T H=X H$ we have that $T H(\{x\})=X H(\{x\})$; thus $\cup M d(x)=\{u \mid x \in$ $N(u)\}$.

Conversely, let $X \subseteq U$. By Lemma 14, we have that $\cup\{\cup M d(x) \mid x \in X\}=\{x \mid N(x) \cap X \neq \emptyset\}$. It follows from Definition 5 that $T H(X)=X H(X)$. We have proved that, $\forall X \subseteq U, T H(X)=X H(X)$. Therefore, $T H=X H$.

By Theorems 15 and 28, we can conclude that $F H=$ $X H \Rightarrow T H=X H$.

In the following, we give a condition under which $T H$ and $V H$ are identical.

Theorem 29. Let $(U, \mathscr{C})$ be a covering approximation space. Then $T H=V H$ if and only if, $\forall x \in U, \cup M d(x)=\cup\{N(u) \mid$ $x \in N(u)\}$.

Proof. We first prove the necessity. $\forall x \in U$, by Definition 5, we have that $T H(\{x\})=\cup M d(x)$ and $V H(\{x\})=\cup\{N(u)$ | $N(u) \cap\{x\} \neq \emptyset\}=\cup\{N(u) \mid x \in N(u)\}$. By the condition $T H=V H$ we have $T H(\{x\})=V H(\{x\})$, and thus $\cup M d(x)=$ $\cup\{N(u) \mid x \in N(u)\}$.

Conversely, let $X \subseteq U$. By Lemma 16, we have that $\cup\{\cup M d(x) \mid x \in X\}=\cup\{N(x) \mid N(x) \cap X \neq \emptyset\}$. It follows from Definition 5 that $T H(X)=V H(X)$. We have proved that, $\forall X \subseteq U, T H(X)=V H(X)$. Therefore, $T H=V H$.

By Theorems 17 and 29, we can conclude that $F H=$ $V H \Rightarrow T H=V H$.

\section{Relationships between $R H$ and $I H, X H$, and $V H$}

In this section, we explore the relationships between the fourth type of covering rough sets and later three types and establish some conditions under which two types of upper approximation operations are identical.

Lemma 30. Let $(U, \mathscr{C})$ be a covering approximation space and $X \subseteq U$. If, $\forall C \in \mathscr{C}, \forall x \in C,\{x\} \in \mathscr{C}$ or $N(x)=C$, then $\cup\{C \mid C \cap(X-C L(X)) \neq \emptyset\}=\cup\{N(x) \mid x \in X-C L(X)\}$.

Proof. $\forall y \in \cup\{C \mid C \cap(X-\mathrm{CL}(X)) \neq \emptyset\}$, there exists $C \in \mathscr{C}$ such that $C \cap(X-\mathrm{CL}(X)) \neq \emptyset$ and $y \in C$. Taking $x \in C \cap(X-$ $\mathrm{CL}(X))$, then $x \in C$ and $x \in X-\mathrm{CL}(X)$. By Lemma 21, we have that $\{x\} \notin \mathscr{C}$. By our condition, this implies that $N(x)=$ $C$. Further, since $x \in X-\mathrm{CL}(X)$, it follows that $C=N(x) \subseteq$ $\cup\{N(x) \mid x \in X-\mathrm{CL}(X)\}$ and so $y \in \cup\{N(x) \mid x \in X-$ $\mathrm{CL}(X)\}$. Therefore,

$\cup\{C \mid C \cap(X-\mathrm{CL}(X)) \neq \emptyset\} \subseteq \cup\{N(x) \mid x \in X-\mathrm{CL}(X)\}$.

On the other hand, $\forall y \in \cup\{N(x) \mid x \in X-\mathrm{CL}(X)\}$, then, $\exists x \in X-\mathrm{CL}(X), y \in N(x)$. By Definition $4, y \in N(x)$ implies that there exists $C \in \mathscr{C}$ such that $x, y \in C$. Clearly, $x \in C \cap$ $(X-\mathrm{CL}(X))$; thus $C \cap(X-\mathrm{CL}(X)) \neq \emptyset$ and so $C \subseteq \cup\{C$ । $C \cap(X-\mathrm{CL}(X)) \neq \emptyset\}$. Thus $y \in \cup\{C \mid C \cap(X-\mathrm{CL}(X)) \neq \emptyset\}$. Therefore,

$\cup\{N(x) \mid x \in X-\mathrm{CL}(X)\} \subseteq \cup\{C \mid C \cap(X-\mathrm{CL}(X)) \neq \emptyset\}$

In summary, $\cup\{C \mid C \cap(X-\mathrm{CL}(X)) \neq \emptyset\}=\cup\{N(x) \mid x \in$ $X-\mathrm{CL}(X)\}$.

In the following, we present a condition under which $\mathrm{RH}$ and $I H$ are identical.

Theorem 31. Let $(U, \mathscr{C})$ be a covering approximation space. Then $R H=I H$ if and only if, $\forall C \in \mathscr{C}, \forall x \in C,\{x\} \in \mathscr{C}$ or $N(x)=C$.

Proof. We first prove the necessity, suppose that there exist $C \in \mathscr{C}$ and $x \in C$ such that $\{x\} \notin \mathscr{C}$ and $N(x) \neq C$; then $N(x) \varsubsetneqq C$. By the definition of CL, $\{x\} \notin \mathscr{C}$ implies that $\mathrm{CL}(\{x\})=\emptyset$; thus by Definition 5, we have that $\operatorname{IH}(\{x\})=$ $N(x)$ and $C \subseteq R H(\{x\})$ (because $C \cap(\{x\}-\operatorname{CL}(\{x\})) \neq \emptyset)$. Since $N(x) \varsubsetneqq C$, it follows that $I H(\{x\}) \varsubsetneqq R H(\{x\})$ and so $I H(\{x\}) \neq R H(\{x\})$, which contradicts the condition $R H=$ $I H$. This completes the proof of the necessity.

Conversely, let $X \subseteq U$. By Lemma 30, we have that $\cup\{C$ | $C \cap(X-\mathrm{CL}(X)) \neq \emptyset\}=\cup\{N(x) \mid x \in X-\mathrm{CL}(X)\}$. It follows from the definitions of $R H$ and $I H$ that $R H(X)=I H(X)$. We have proved that, $\forall X \subseteq U, R H(X)=I H(X)$. Therefore, $R H=I H$.

Proposition 32. Let $(U, \mathscr{C})$ be a covering approximation space. If $R H=I H$, then $T H=R H$.

Proof. By Theorem 23, we only need to prove that, $\forall C_{1}, C_{2} \in$ $\mathscr{C}, C_{1} \neq C_{2}$, and $x \in C_{1} \cap C_{2},\{x\} \in \mathscr{C}$. Suppose that $\{x\} \notin \mathscr{C}$, then by $R H=I H$ and Theorem 31, it follows from $x \in C_{1}$ and $x \in C_{2}$ that $N(x)=C_{1}$ and $N(x)=C_{2}$. Thus $C_{1}=C_{2}$, a contradiction with $C_{1} \neq C_{2}$. This completes the proof. 
Lemma 33. Let $(U, \mathscr{C})$ be a covering approximation space, $C \in$ $\mathscr{C}$, and $y \in C$. If $\{y\} \in \mathscr{C}$ and $R H=X H$, then $\forall x \in C$, $\{x\} \in \mathscr{C}$.

Proof. Suppose that, $\exists x \in C,\{x\} \notin \mathscr{C}$, then by $\{y\} \in \mathscr{C}$, we have that $x \neq y$. Since $\{x\} \notin \mathscr{C}$, it follows that $\operatorname{CL}(\{x\})=\emptyset$. Thus $x \in C \cap(\{x\}-\operatorname{CL}(\{x\})) \neq \emptyset$ and so by the definition of $R H$, we get that $C \subseteq R H(\{x\})$. Hence $y \in R H(\{x\})$. On the other hand, by $\{y\} \in \mathscr{C}$, we have that $N(y)=\{y\}$, and by $x \neq y$, we can conclude that $N(y) \cap\{x\}=\emptyset$. It follows from the definition of $X H$ that $y \notin X H(\{x\})$. Thus, $R H(\{x\}) \neq$ $X H(\{x\})$, which is a contradiction with the condition $R H=$ $X H$. Therefore, $\forall x \in C,\{x\} \in \mathscr{C}$.

Lemma 34. Let $(U, \mathscr{C})$ be a covering approximation space and $X \subseteq U$. If $\forall C \in \mathscr{C}$, we have that $\forall x \in C, N(x)=C$ or $\forall x \in C$, $\{x\} \in \mathscr{C}$, then the following assertions hold.

(1) $C L(X)=\{x \mid N(x) \cap C L(X) \neq \emptyset\}$.

(2) $\cup\{C \mid C \cap(X-C L(X)) \neq \emptyset\}=\{x \mid N(x) \cap(X-$ $C L(X)) \neq \emptyset\}$.

Proof. (1) We only need to prove that $\mathscr{C}$ satisfies the condition of Lemma 13. $\forall C \in \mathscr{C}, \forall u \in U \backslash C$, we will show that $N(u) \cap$ $C=\emptyset$. It is clear that, $\exists K \in \mathscr{C}, N(u) \subseteq K$. If $\{u\} \in \mathscr{C}$, then $N(u)=\{u\}$. By $u \in U \backslash C$, we have that $N(u) \cap C=\emptyset$. If $\{u\} \notin \mathscr{C}$, then, by $u \in K$ and the assumed condition, we can conclude that, $\forall x \in K, N(x)=K$; thus $N(u)=K$. Suppose that $N(u) \cap C \neq \emptyset$; then $K \cap C \neq \emptyset$. Taking $x \in K \cap C$, then $K=N(x) \subseteq C$ and so $u \in C$. This is a contradiction with our choice, $u \in U \backslash C$. Hence, $N(u) \cap C=\emptyset$. We have proved that $\mathscr{C}$ satisfies the condition of Lemma 13. Therefore, by Lemma 13 , we get that $\mathrm{CL}(X)=\{x \mid N(x) \cap \mathrm{CL}(X) \neq \emptyset\}$.

(2) $\forall y \in \cup\{C \mid C \cap(X-\mathrm{CL}(X)) \neq \emptyset\}$, there exists $C \in \mathscr{C}$ such that $C \cap(X-\mathrm{CL}(X)) \neq \emptyset$ and $y \in C$. Taking $z \in C \cap(X-$ $\mathrm{CL}(X))$, then $z \in C$ and $z \in X-\mathrm{CL}(X)$. By Lemma $21,\{z\} \notin$ $\mathscr{C}$; hence by the assumed condition, we have that, $\forall x \in C$, $N(x)=C$, and so $N(y)=C$. It follows by $C \cap(X-\mathrm{CL}(X)) \neq \emptyset$ that $N(y) \cap(X-\mathrm{CL}(X)) \neq \emptyset$ and thus, $y \in\{x \mid N(x) \cap(X-$ $\mathrm{CL}(X)) \neq \emptyset\}$. Therefore,

$$
\begin{aligned}
& \cup\{C \mid C \cap(X-\mathrm{CL}(X)) \neq \emptyset\} \\
& \quad \subseteq\{x \mid N(x) \cap(X-\mathrm{CL}(X)) \neq \emptyset\} .
\end{aligned}
$$

On the other hand, $\forall y \in\{x \mid N(x) \cap(X-\mathrm{CL}(X)) \neq \emptyset\}$, then $N(y) \cap(X-\mathrm{CL}(X)) \neq \emptyset$. By Definition $4, \exists C \in \mathscr{C}$, $N(y) \subseteq C$; thus $y \in C$ and $C \cap(X-\operatorname{CL}(X)) \neq \emptyset$. It follows that $y \in \cup\{C \mid C \cap(X-\mathrm{CL}(X)) \neq \emptyset\}$. Therefore,

$$
\begin{aligned}
& \{x \mid N(x) \cap(X-\mathrm{CL}(X)) \neq \emptyset\} \\
& \quad \subseteq \cup\{C \mid C \cap(X-\mathrm{CL}(X)) \neq \emptyset\} .
\end{aligned}
$$

In summary, $\cup\{C \mid C \cap(X-\mathrm{CL}(X)) \neq \emptyset\}=\{x \mid N(x) \cap(X-$ $\mathrm{CL}(X)) \neq \emptyset\}$.

In the following, we present a condition under which $R H$ and $X H$ are identical.
Theorem 35. Let $(U, \mathscr{C})$ be a covering approximation space. Then $R H=X H$ if and only if, $\forall C \in \mathscr{C}$, we have that $\forall x \in C$, $N(x)=C$ or $\forall x \in C,\{x\} \in \mathscr{C}$.

Proof. We first prove the necessity. Let $C \in \mathscr{C}$ and $y \in C$. If $\{y\} \in \mathscr{C}$, then by Lemma 33, we have that, $\forall x \in C,\{x\} \in \mathscr{C}$. The necessity holds. If $\{y\} \notin \mathscr{C}$, then we shall show that $\forall x \in$ $C, N(x)=C$. Suppose that $N(x) \neq C$, then by Definition 4 , $N(x) \varsubsetneqq C$. Taking $w \in C-N(x)$, then $N(x) \cap\{w\}=\emptyset$; thus by the definition of $X H, x \notin X H(\{w\})$. On the other hand, suppose that $\{w\} \in \mathscr{C}$; then by Lemma 33, we conclude that $\{y\} \in \mathscr{C}$, a contradiction. Hence $\{w\} \notin \mathscr{C}$ and so $\operatorname{CL}(\{w\})=\emptyset$. It follows that $w \in C \cap(\{w\}-\operatorname{CL}(\{w\})) \neq \emptyset$, which implies that $C \subseteq R H(\{w\})$ by the definition of $R H$. Hence $x \in R H(\{w\})$. Thus $R H(\{w\}) \neq X H(\{w\})$, which contradicts the condition, $R H=X H$. Therefore, $\forall x \in C, N(x)=C$. This finishes the proof of the necessity.

Conversely, let $X \subseteq U$. By Lemma 34, we obtain that $\mathrm{CL}(X)=\{x \mid N(x) \cap \mathrm{CL}(X) \neq \emptyset\}$ and $\cup\{C \mid \mathrm{C} \cap(X-\mathrm{CL}(X)) \neq$ $\emptyset\}=\{x \mid N(x) \cap(X-\mathrm{CL}(X)) \neq \emptyset\}$. Thus

$$
\begin{aligned}
R H(X)= & \mathrm{CL}(X) \cup(\cup\{C \mid C \cap(X-\mathrm{CL}(X)) \neq \emptyset\}) \\
= & \{x \mid N(x) \cap \mathrm{CL}(X) \neq \emptyset\} \\
& \cup(\{x \mid N(x) \cap(X-\mathrm{CL}(X)) \neq \emptyset\}) \\
= & \{x \mid N(x) \cap X \neq \emptyset\}=X H(X) .
\end{aligned}
$$

We have proved that, $\forall X \subseteq U, R H(X)=X H(X)$. Therefore, $R H=X H$.

Proposition 36. Let $(U, \mathscr{C})$ be a covering approximation space. If $R H=X H$, then $R H=I H$.

Proof. The proof follows directly from Theorems 31 and 35.

By Propositions 32 and 36, we conclude that $R H=X H \Rightarrow$ $R H=I H \Rightarrow T H=R H$.

In the following, we present a condition under which $R H$ and $\mathrm{VH}$ are identical.

Theorem 37. Let $(U, \mathscr{C})$ be a covering approximation space. Then $R H=V H$ if and only if $\mathscr{C}$ satisfies the following conditions: (1) $\forall C \in \mathscr{C}, \forall u \in U \backslash C$, or $N(u) \cap C=\emptyset$; (2) $\forall C \in$ $\mathscr{C}, \forall x \in C,\{x\} \in \mathscr{C}$, or $N(x)=C$.

Proof. We first prove the necessity.

(1) We will prove that, $\forall C \in \mathscr{C}, \forall u \in U \backslash C, N(u) \cap C=\emptyset$. Let $C \in \mathscr{C}$. Then $R H(C)=C$ and $V H(C)=\bigcup\{N(x) \mid N(x) \cap$ $C \neq \emptyset\}$. By $R H=V H$, we get that $R H(C)=V H(C)$ and so $C=\cup\{N(x) \mid N(x) \cap C \neq \emptyset\} . \forall u \in U \backslash C$, if $N(u) \cap C \neq \emptyset$, then $N(u) \subseteq \cup\{N(x) \mid N(x) \cap C \neq \emptyset\}$ and so $u \in \cup\{N(x) \mid$ $N(x) \cap C \neq \emptyset\}$. By $u \in U \backslash C$, we have that $u \notin C$. Therefore, $C \neq$ $\cup\{N(x) \mid N(x) \cap C \neq \emptyset\}$, a contradiction. Thus, $N(u) \cap C=\emptyset$.

(2) We will show that, $\forall C \in \mathscr{C}, \forall x \in C,\{x\} \in \mathscr{C}$ or $N(x)=C$. Suppose that, $\exists C \in \mathscr{C}, \exists x_{0} \in C,\left\{x_{0}\right\} \notin \mathscr{C}$ and $N\left(x_{0}\right) \neq C .\left\{x_{0}\right\} \notin \mathscr{C}$ implies that $\operatorname{CL}\left(\left\{x_{0}\right\}\right)=\emptyset$; thus $C \cap$ 
$\left(\left\{x_{0}\right\}-\mathrm{CL}\left(\left\{x_{0}\right\}\right)\right) \neq \emptyset$ and so $C \subseteq R H\left(\left\{x_{0}\right\}\right)$. By $R H=V H$, we get that $R H\left(\left\{x_{0}\right\}\right)=V H\left(\left\{x_{0}\right\}\right)$. Thus,

$$
C \subseteq V H\left(\left\{x_{0}\right\}\right)=\cup\left\{N(x) \mid N(x) \cap\left\{x_{0}\right\} \neq \emptyset\right\} .
$$

On the other hand, by $R H=V H$, we have that $R H(C)=$ $V H(C)$ and so $C=\cup\{N(x) \mid N(x) \cap C \neq \emptyset\}$. It follows from $x_{0} \in C$ that

$$
\begin{aligned}
\cup & \left\{N(x) \mid N(x) \cap\left\{x_{0}\right\} \neq \emptyset\right\} \\
& \subseteq \cup\{N(x) \mid N(x) \cap C \neq \emptyset\}=C .
\end{aligned}
$$

In summary, $C=\cup\left\{N(x) \mid N(x) \cap\left\{x_{0}\right\} \neq \emptyset\right\}$. In addition, since $N\left(x_{0}\right) \neq C$, it follows from Definition 4 that, $\exists K \in \mathscr{C}$, $x_{0} \in K$ and $C \nsubseteq K . x_{0} \in K$ implies that $\cup\{N(x) \mid N(x) \cap$ $\left.\left\{x_{0}\right\} \neq \emptyset\right\} \subseteq V H(K)$; thus $C \subseteq V H(K)$. Clearly, $R H(K)=K$. By $R H=V H$, we get that $R H(K)=V H(K)$. Thus, $C \subseteq K$, a contradiction with $C \nsubseteq K$. Therefore, $\forall C \in \mathscr{C}, \forall x \in C$, $\{x\} \in \mathscr{C}$ or $N(x)=C$.

By (1) and (2), the necessity holds.

Conversely, let $X \subseteq U$. We will show that $\cup\{C \mid C \cap(X-$ $\mathrm{CL}(X)) \neq \emptyset\}=\cup\{N(x) \mid N(x) \cap(X-\mathrm{CL}(X)) \neq \emptyset\} . \forall y \in \cup\{C \mid$ $C \cap(X-\mathrm{CL}(X)) \neq \emptyset\}$, then, $\exists C \in \mathscr{C}, C \cap(X-\mathrm{CL}(X)) \neq \emptyset$, and $y \in C$. Taking $u \in C \cap(X-\operatorname{CL}(X))$, then $u \in C$ and $u \in X-\mathrm{CL}(X)$. By Lemma $21,\{u\} \notin \mathscr{C}$, and by the condition (2), $N(u)=C$. Thus $y \in N(u)$ and $N(u) \cap(X-\mathrm{CL}(X)) \neq \emptyset$, and so $y \in \cup\{N(x) \mid N(x) \cap(X-\mathrm{CL}(X)) \neq \emptyset\}$. Thus,

$$
\begin{aligned}
& \cup\{C \mid C \cap(X-\mathrm{CL}(X)) \neq \emptyset\} \\
& \quad \subseteq \cup\{N(x) \mid N(x) \cap(X-\mathrm{CL}(X)) \neq \emptyset\} .
\end{aligned}
$$

On the other hand, $\forall y \in \cup\{N(x) \mid N(x) \cap(X-\mathrm{CL}(X)) \neq \emptyset\}$, then, $\exists x \in U, N(x) \cap(X-\mathrm{CL}(X)) \neq \emptyset$ and $y \in N(x)$. It is clear that, $\exists C \in \mathscr{C}, N(x) \subseteq C$. Thus $y \in C$ and $C \cap(X-\mathrm{CL}(X)) \neq \emptyset$ and so $y \in \cup\{C \mid C \cap(X-\mathrm{CL}(X)) \neq \emptyset\}$. Hence,

$$
\begin{gathered}
\cup\{N(x) \mid N(x) \cap(X-\mathrm{CL}(X)) \neq \emptyset\} \\
\subseteq \cup\{C \mid C \cap(X-\mathrm{CL}(X)) \neq \emptyset\} .
\end{gathered}
$$

In summary, $\cup\{C \mid C \cap(X-\mathrm{CL}(X)) \neq \emptyset\}=\cup\{N(x) \mid N(x) \cap$ $(X-\mathrm{CL}(X)) \neq \emptyset\}$. Further, by Lemma 13, we can get that $\mathrm{CL}(X)=\cup\{N(x) \mid N(x) \cap \mathrm{CL}(X) \neq \emptyset\}$. Thus,

$$
\begin{aligned}
R H(X)= & \operatorname{CL}(X) \cup(\cup\{C \mid C \cap(X-\operatorname{CL}(X)) \neq \emptyset\}) \\
= & \cup\{N(x) \mid N(x) \cap \operatorname{CL}(X) \neq \emptyset\} \\
& \cup(\cup\{N(x) \mid N(x) \cap(X-\operatorname{CL}(X)) \neq \emptyset\}) \\
= & \cup\{N(x) \mid N(x) \cap X \neq \emptyset\}=V H(X) .
\end{aligned}
$$

We have proved that, $\forall X \subseteq U, R H(X)=V H(X)$. Therefore, $R H=V H$.

\section{Relationships among $I H, X H$, and $V H$}

In this section, we will study the relationships among the last three types of covering rough sets, and establish the conditions under which two types of upper approximation operations are identical.
Theorem 38. Let $(U, \mathscr{C})$ be a covering approximation space. Then the following conditions are equivalent.
(1) $\forall X \subseteq U, I H(X)=X H(X)$.
(2) $\forall X \subseteq U, I H(X)=V H(X)$.
(3) $\forall X \subseteq U, X H(X)=V H(X)$.
(4) $\{N(x) \mid x \in U\}$ is a partition.

Proof. (1) $\Rightarrow$ (4) Suppose that $\{N(x) \mid x \in U\}$ is not a partition; then there exist $x, y \in U$ such that $N(x) \neq N(y)$ and $N(x) \cap N(y) \neq \emptyset$. We will show that $y \notin N(x)$ or $x \notin N(y)$. Otherwise, by Definition 4,y $\in N(x)$ implies that $N(y) \subseteq N(x)$, and $x \in N(y)$ implies $N(x) \subseteq N(y)$. Thus, $N(x)=N(y)$, a contradiction. Hence, $y \notin N(x)$ or $x \notin N(y)$. Without loss of generality, we assume that $y \notin N(x)$. Taking $z \in N(x) \cap N(y)$, we have $N(z) \subseteq N(x)$ and $N(z) \subseteq N(y)$. Hence, $y \notin N(x)$ implies that $y \notin N(z)$. Then by the definition of $I H$, we get that $I H(\{z\})=N(z)$ and so $y \notin I H(\{z\})$. Since $N(y) \cap\{z\}=\{z\} \neq \emptyset$, it follows from the definition of $X H$ that $y \in X H(\{z\})$. Thus, $I H(\{z\}) \neq$ $X H(\{z\})$, which is a contradiction with the condition, $I H=$ $X H$. Hence $\{N(x) \mid x \in U\}$ is a partition.

$(2) \Rightarrow(4)$ The proof is similar to that of $(1) \Rightarrow(4)$.

(3) $\Rightarrow$ (4) Suppose that $\{N(x) \mid x \in U\}$ is not a partition; then there exist $x, y \in U$ such that $N(x) \neq N(y)$ and $N(x) \cap$ $N(y) \neq \emptyset$. By the proof of $(1) \Rightarrow(4)$, we have that $y \notin N(x)$ or $x \notin N(y)$. Without loss of generality, we assume that $y \notin$ $N(x)$. Taking $z \in N(x) \cap N(y)$, we have $N(z) \subseteq N(x)$ and $N(z) \subseteq N(y)$. Hence $y \notin N(x)$ implies that $y \notin N(z)$. Then by Definition 5, we get that $z \notin X H(\{y\})$ (because $N(z) \cap$ $\{y\}=\emptyset)$ and $z \in N(y) \subseteq V H(\{y\})$ (because $N(y) \cap\{y\}=$ $\{y\} \neq \emptyset)$. Thus, $X H(\{y\}) \neq V H(\{y\})$, which is a contradiction with the condition $X H=V H$. Hence $\{N(x) \mid x \in U\}$ is a partition.

$(4) \Rightarrow(1),(2),(3)$ It is obvious.

\section{Conclusions}

This paper discusses the relationships among seven pairs of common approximation operators; that is to say, the paper investigates the connections of seven types of covering rough set models. These studies illustrate that we can induce different covering rough set models from a covering, but they may be the same model when the covering meets some conditions. It is meaningful to establish a unified framework for covering rough set models and help a decision maker to choose a suitable rough set model for data analysis.

\section{Conflict of Interests}

The author declares that there is no conflict of interests regarding the publication of this paper.

\section{Acknowledgments}

The author thanks the anonymous reviewers and the editor for their constructive and valuable comments. This work is supported by the Natural Science Foundation of Shanxi 
Province (no. 2012011011-1), the Nature Science Foundation of Shanxi Province (no. 2008011012), and the Foundation of Shanxi Normal University (Grant no. 872022).

\section{References}

[1] Z. Pawlak, "Rough sets," International Journal of Computer and Information Sciences, vol. 11, no. 5, pp. 341-356, 1982.

[2] Z. Pawlak, Rough Sets: Theoretical Aspects of Reasoning about Data, Kluwer Academic Publishers, Boston, Mass, USA, 1991.

[3] Z. Bonikowski, E. Bryniarski, and U. Wybraniec-Skardowska, "Extensions and intentions in the rough set theory," Information Sciences, vol. 107, no. 1-4, pp. 149-167, 1998.

[4] G. Liu, "The axiomatization of the rough set upper approximation operations," Fundamenta Informaticae, vol. 69, no. 3, pp. 331-342, 2006.

[5] Z. Pawlak and A. Skowron, "Rudiments of rough sets," Information Sciences, vol. 177, no. 1, pp. 3-27, 2007.

[6] J. A. Pomykała, "Approximation operations in approximation space," Bulletin of the Polish Academy of Sciences, vol. 35, no. 910, pp. 653-662, 1987.

[7] Y. Y. Yao, "Constructive and algebraic methods of the theory of rough sets," Information Sciences, vol. 109, no. 1-4, pp. 21-47, 1998.

[8] W. Zakowski, "Approximations in the space $(U, \Pi)$," Demonstratio Mathematica, vol. 16, no. 3, pp. 761-769, 1983.

[9] H. Zhang, H. Liang, and D. Liu, "Two new operators in rough set theory with applications to fuzzy sets," Information Sciences, vol. 166, no. 1-4, pp. 147-165, 2004.

[10] W. Zhu, "Relationship among basic concepts in covering-based rough sets," Information Sciences, vol. 179, no. 14, pp. 2478-2486, 2009.

[11] W. Zhu and F. Wang, "Reduction and axiomization of covering generalized rough sets," Information Sciences, vol. 152, pp. 217230, 2003.

[12] T. Y. Lin and N. Cercone, Eds., Rough Sets and Data Mining, Kluwer Academic Publishers, Boston, Mass, USA, 1997.

[13] L. Polkowski and A. Skowron, Eds., Rough Sets and Current Trends in Computing, vol. 1424, Springer, Berlin, Germany, 1998.

[14] L. Polkowski and A. Skowron, Eds., Rough Sets in Knowledge Discovery, vol. 1-2, Physica, Heidelberg, Germany, 1998.

[15] N. Zhong, Y. Yao, and M. Ohshima, "Peculiarity oriented multidatabase mining," IEEE Transactions on Knowledge and Data Engineering, vol. 15, no. 4, pp. 952-960, 2003.

[16] W. Zhu and F. Y. Wang, "Covering based granular computing for conflict analysis," in Proceedings of the IEEE International Conference on Intelligence and Security Informatics (ISI '06), vol. 3975 of Lecture Notes in Computer Science, pp. 566-571, San Diego, Calif, USA, May 2006.

[17] W. Ziarko, Ed., Rough Sets, Fuzzy Sets, and Knowledge Discovery, Springer, Berlin, Germany, 1994.

[18] R. Slowinski and D. Vanderpooten, "A generalized definition of rough approximations based on similarity," IEEE Transactions on Knowledge and Data Engineering, vol. 12, no. 2, pp. 331-336, 2000.

[19] G. Cattaneo and D. Ciucci, "Algebraic structures for rough sets," in Transactions on Rough Sets II, vol. 3135 of Lecture Notes in Computer Science, pp. 208-252, 2005.

[20] Z. Meng and Z. Shi, "A fast approach to attribute reduction in incomplete decision systems with tolerance relation-based rough sets," Information Sciences, vol. 179, no. 16, pp. 2774-2793, 2009.

[21] A. Skowron and J. Stepaniuk, "Tolerance approximation spaces," Fundamenta Informaticae, vol. 27, no. 2-3, pp. 245-253, 1996.

[22] M. Kondo, "On the structure of generalized rough sets," Information Sciences, vol. 176, no. 5, pp. 589-600, 2006.

[23] G. Liu and W. Zhu, "The algebraic structures of generalized rough set theory," Information Sciences, vol. 178, no. 21, pp. 41054113, 2008.

[24] Y. Y. Yao, "Relational interpretations of neighborhood operators and rough set approximation operators," Information Sciences, vol. 111, no. 1-4, pp. 239-259, 1998.

[25] W. Zhu, "Generalized rough sets based on relations," Information Sciences, vol. 177, no. 22, pp. 4997-5011, 2007.

[26] E. Bryniarski, "A calculus of rough sets of the first order," Bulletin of the Polish Academy of Sciences: Mathematics, vol. 37, no. 1-6, pp. 71-77, 1989.

[27] T. Yang and Q. Li, "Reduction about approximation spaces of covering generalized rough sets," International Journal of Approximate Reasoning, vol. 51, no. 3, pp. 335-345, 2010.

[28] E. C. C. Tsang, D. Chen, J. W. T. Lee, and D. S. Yeung, "On the upper approximations of covering generalized rough sets," in Proceedings of the 3rd International Conference on Machine Learning and Cybernetics, pp. 4200-4203, August 2004.

[29] W. Zhu, "Relationship between generalized rough sets based on binary relation and covering," Information Sciences, vol. 179, no. 3, pp. 210-225, 2009.

[30] W. Zhu and F. Y. Wang, "A new type of covering rough sets," in Proceedings of the 3rd International IEEE Conference on Intelligent Systems, pp. 444-449, London, UK, September 2006.

[31] W. Xu and W. Zhang, "Measuring roughness of generalized rough sets induced by a covering," Fuzzy Sets and Systems, vol. 158, no. 22, pp. 2443-2455, 2007.

[32] Z. Y. Xu and Q. Wang, "Properties of covering rough sets model," Journal of Henan Normal University, vol. 33, no. 1, pp. 130-132, 2005.

[33] W. Zhu and F.-Y. Wang, "Topological properties in coveringbased rough sets," in Proceedings of the 4th International Conference on Fuzzy Systems and Knowledge Discovery (FSKD '07), J. Lei, J. Yu, and S. G. Zhou, Eds., vol. 1, pp. 289-293, Haikou, China, August 2007.

[34] M. Restrepo, C. Cornelis, and J. Gómez, "Duality, conjugacy and adjointness of approximation operators in covering-based rough sets," International Journal of Approximate Reasoning, vol. 55, no. 1, part 4, pp. 469-485, 2014.

[35] W. Zhu and F. Wang, "On three types of covering-based rough sets," IEEE Transactions on Knowledge and Data Engineering, vol. 19, no. 8, pp. 1131-1144, 2007. 


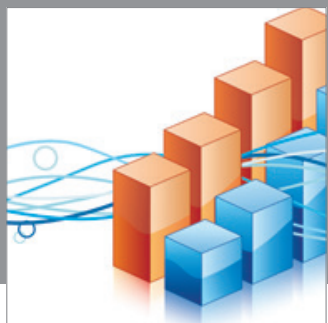

Advances in

Operations Research

mansans

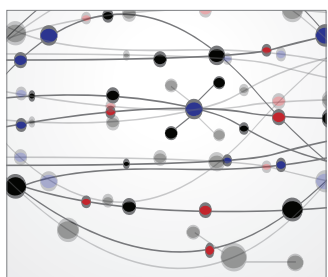

The Scientific World Journal
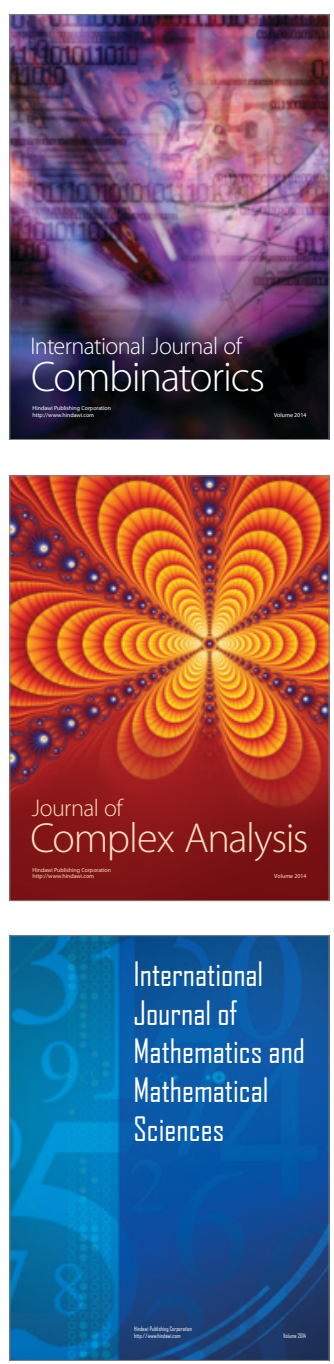
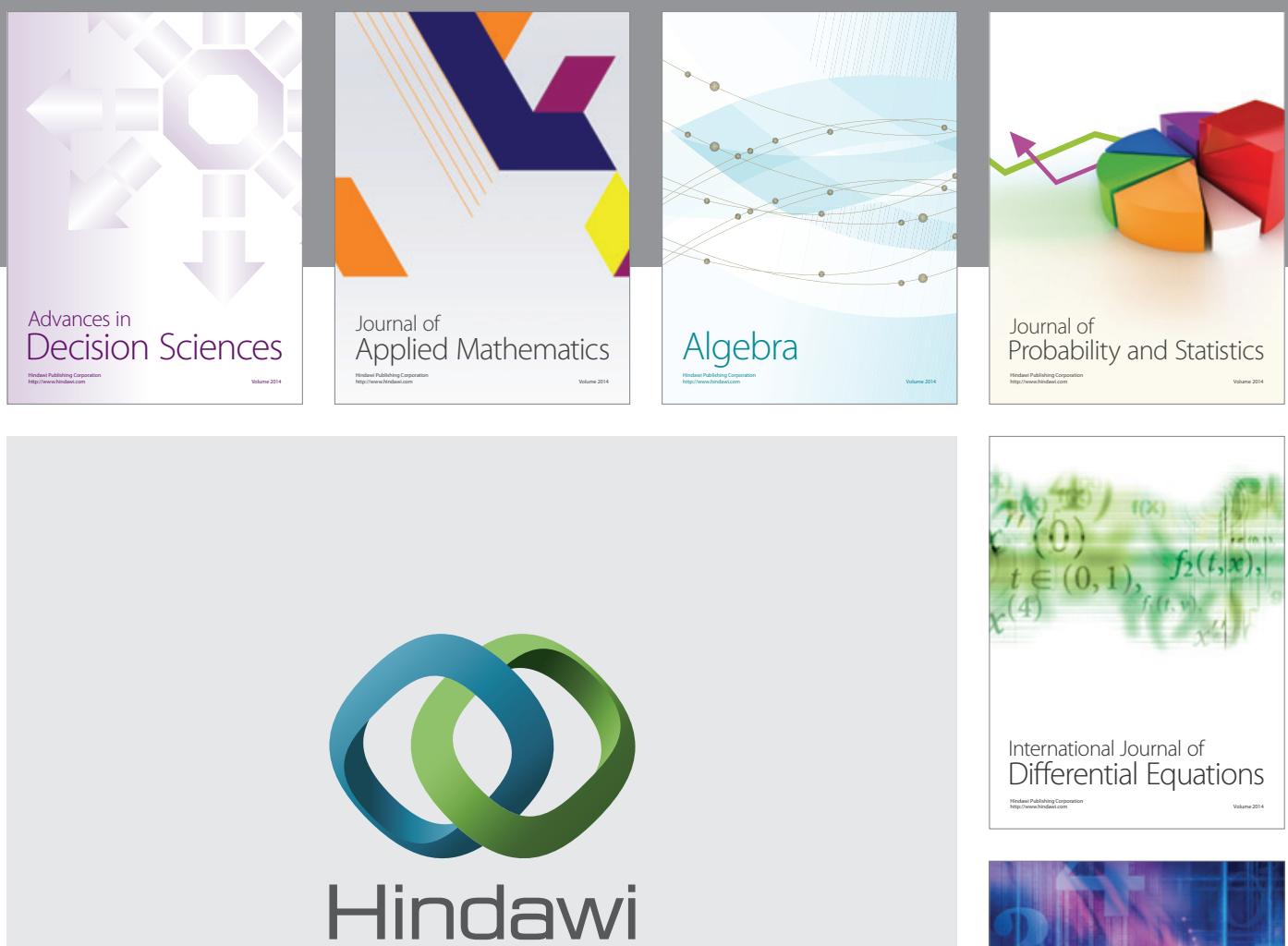

Submit your manuscripts at http://www.hindawi.com
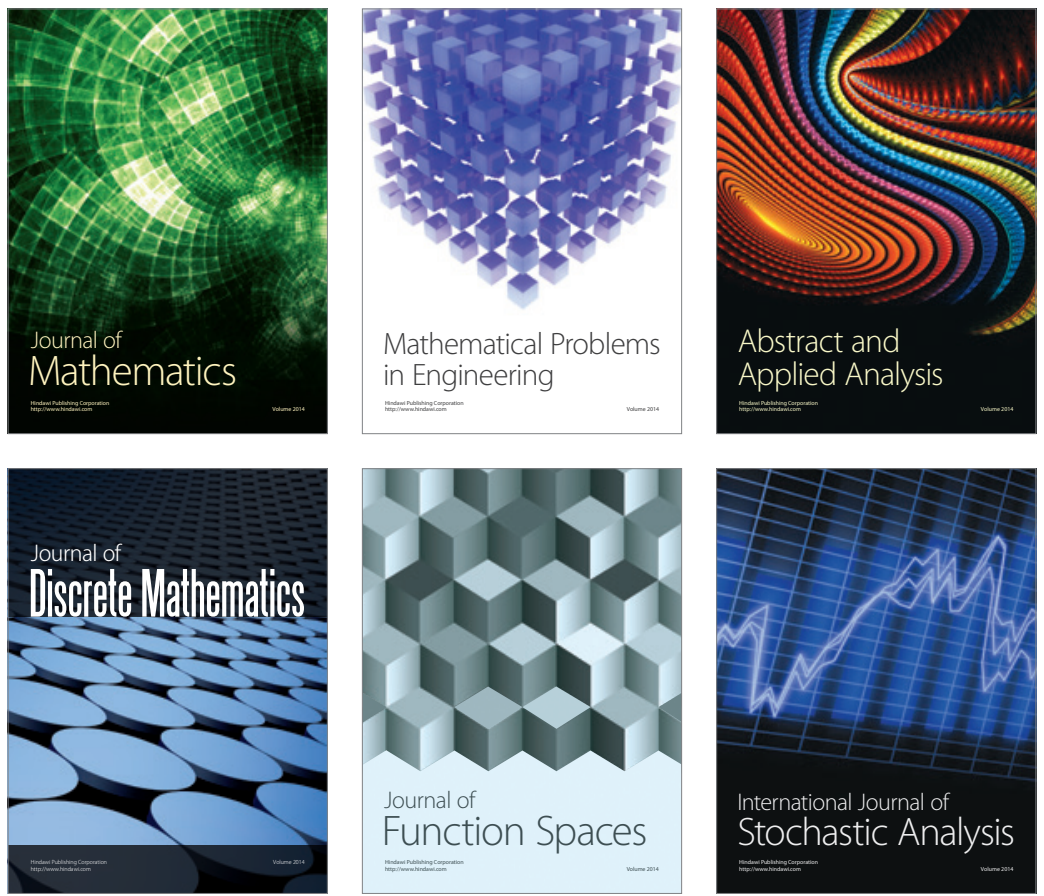

Journal of

Function Spaces

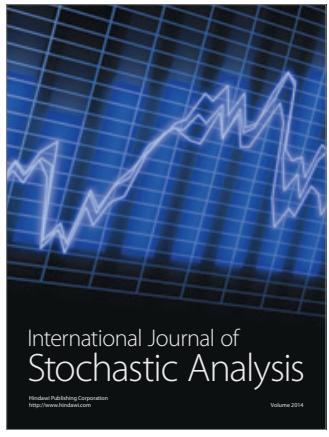

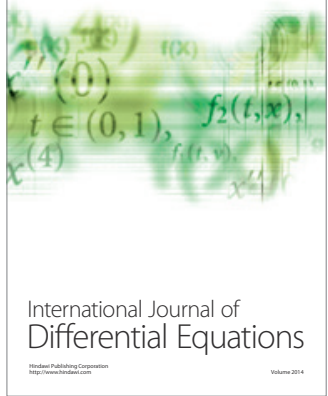
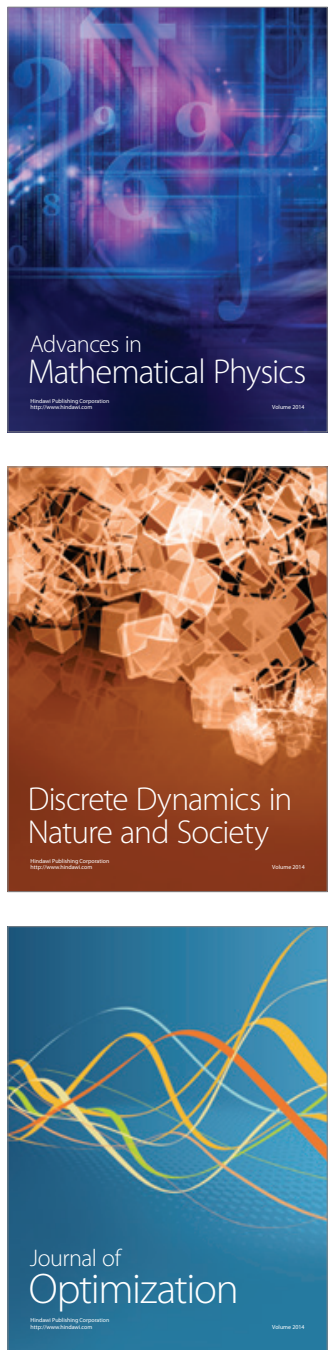